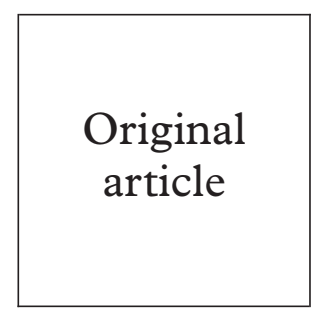

Department of Neuroimmunology, Institute of Neurology, National Hospital for Neurology and Neurosurgery, Queen Square, London WC1N 3BG, UK

A J E Green

G Giovannoni

E J Thompson

MRI Unit, Department of Imaging, University College London Hospitals, Middlesex

Hospital Site, London W1N 8AA, UK

M A Hall-Craggs

Department of Sexually Transmitted Diseases, Royal Free and University College Medical School, University College London and Mortimer Market Centre, Camden and Islington Community Health

Services Trust, London WC1E 6AU, UK

R F Miller

Correspondence to: Dr R Miller, Department of STD, RFUCMS, Mortimer Market Centre, Off Capper Street, London WC1E 6AU, UK

rmiller@gum.ucl.ac.uk

Accepted for publication 29 June 2000

\title{
Cerebrospinal fluid tau concentrations in HIV infected patients with suspected neurological disease
}

\author{
Alison J E Green, Gavin Giovannoni, Margaret A Hall-Craggs, Ed J Thompson,
} Robert F Miller

Objectives: To measure cerebrospinal fluid (CSF) tau in HIV infected patients with acute neurological episodes and to correlate the findings with the type and severity of neurological disease.

Methods: CSF tau was prospectively measured in 76 consecutive HIV infected patients admitted to a specialist unit at UCL Hospitals, London, for investigation of acute neurological episodes: the results were compared with the clinical diagnoses.

Results: 24 patients had HIV associated dementia complex (HADC), 10 had lymphoma (including four with primary CNS lymphoma), 20 had cerebral infections (including five with CMV encephalitis, five with VZV infection, seven with cryptococcal meningitis, two with toxoplasmosis, and one with progressive multifocal leucoencephalopathy); 22 patients had miscellaneous conditions, including nine with self limiting headache/fever. 62 patients $(82 \%)$ had normal CSF tau concentration and 14 patients (18\%) had elevated tau. In those with HADC, there was no correlation between the degree of dementia or atrophy on magnetic resonance imaging and CSF tau. Elevated CSF tau was associated with poor outcome as six of eight patients who died within 4 weeks of lumbar puncture had elevated tau $(p=0.0024$, two tailed Fisher's exact test).

Conclusions: CSF tau levels are not elevated in the majority of HIV infected patients presenting with acute neurological episodes. CSF tau levels show no correlation with severity of dementia/ atrophy on magnetic resonance imaging. Although elevated CSF tau was observed in some patients with conditions causing cerebral necrosis, the finding did not delineate underlying pathology but was associated with poor outcome.

(Sex Transm Inf 2000;76:443-446)

Keywords: tau; AIDS; cerebrospinal fluid; dementia; magnetic resonance imaging

\section{Introduction}

Tau protein is a microtubule associated phosphoprotein which promotes microtubule assembly and stabilisation. ${ }^{1}$ It is found in high concentrations within axons of the central nervous system ${ }^{1}$ and elevated concentrations of tau may be detected in cerebrospinal fluid of patients with neuronal damage due to acute ischaemic stroke ${ }^{2}$ and Creutzfeldt-Jakob disease. $^{23}$ CSF tau concentrations are also elevated in patients with Alzheimer's disease ${ }^{24}$ and frontotemporal dementia. ${ }^{5}$ Elevated tau is thought to be related to neuronal loss, which occurs in these conditions. By contrast elevations of CSF tau are not seen in all neurodegenerative disorders-for example, Parkinson's disease, but this may simply reflect the extent and rate of neurodegeneration.

Clinically apparent neurological dysfunction occurs in up to $40 \%$ of patients with AIDS, and at necropsy, over $75 \%$ have abnormalities of the central nervous system. Cerebral infection with HIV itself produces a subacute dementia known as HIV associated dementia complex (HADC). Neuropathological changes associated with HADC include a giant cell encephalitis, leucoencephalopathy with myelin pallor, astrogliosis, and neuronal loss. HIV viral load, macrophage activation markers and tumour necrosis factor $\alpha(\mathrm{TNF}-\alpha)$ levels in the brain correlate with the presence and severity of dementia ${ }^{6}$ whereas cortical neuronal counts do not. ${ }^{7}$ Tangle-like structures showing immunoreactivity to tau have been described in brains of HIV infected patients, ${ }^{8}$ yet measurements of tau in CSF of this patient group have shown conflicting results. In one study CSF tau levels were normal in $32 \mathrm{HIV}$ positive patients who were either neurologically asymptomatic or had neurocognitive disorders or peripheral neuropathy. ${ }^{9}$ By contrast, CSF tau was elevated in three of four HIV infected patients with unspecified neurological diagnoses. ${ }^{2}$ In this prospective study we measured CSF tau in consecutive HIV positive patients undergoing diagnostic lumbar puncture for investigation of suspected neurological disease. In those with HADC we attempted to correlate CSF tau measurements with the clinical severity of dementia, and as a marker of neuronal loss, the degree of atrophy on cranial magnetic resonance imaging.

\section{Methods}

We prospectively studied 76 consecutive HIV-1 antibody positive patients admitted to a specialist HIV/AIDS inpatient unit at University College Hospitals (Middlesex Hospital site) for investigation of neurological episodes. 
The study was carried out within the guidelines of the Middlesex Hospital clinical investigations panel.

The patients were aged $26-72$ years (median 37 years). Sixty two patients were males (58 white homosexuals, four African heterosexuals) and 14 were females (11 African and three white), 13 of whom were heterosexuals and one whose risk factor was a "needlestick" injury. As a group the patients were profoundly immunosuppressed with CD4 + T lymphocyte counts ranging from 0 to 440 cells $\times 10^{6} / 1$ (median 40 cells $\times 10^{6} / 1$, normal range $250-1200$ cells $\times$ $\left.10^{6} / 1\right)$. All but seven patients had CD $4+\mathrm{T}$ lymphocyte counts $\leqslant 250$ cells $\times 10^{6} / 1$.

All patients were under the care of a specialist HIV physician. They were investigated using a unit protocol. Following clinical assessment and computed tomography (CT) and/or magnetic resonance imaging (MRI) of the brain, lumbar puncture was carried out. In addition to routine biochemical analyses, CSF was stained histochemically with Gram's stain, auramine, Grocott's methenamine silver, India ink, and mucicarmine and cultured for bacteria, mycobacteria, and fungi. Assays for cryptococcal antigen and tests for antibodies to Treponema pallidum and Toxoplasma gondii were carried out. CSF was also analysed by nested polymerase chain reaction (nPCR) for presence of cytomegalovirus (CMV), varicella zoster virus (VZV), herpes simplex virus type-1 and type-2, Epstein-Barr virus and JC virus as previously described. ${ }^{10}$ An aliquot of CSF (250 $\mu \mathrm{l})$ was frozen immediately at $-20^{\circ} \mathrm{C}$ and stored for subsequent tau measurement. A diagnosis of HADC was made on the basis of presentation with a subacute onset, with at least 1 month of cognitive deficit with decline in motivation, emotional control, or change in social behaviour in the absence of clouding of consciousness, ${ }^{11}$ and in addition the absence of demonstrable infection of the CSF by staining, culture, and nPCR. The clinical severity of the cognitive deficit was recorded using the Memorial Sloan-Kettering criteria. ${ }^{12}$ Cranial MR images of those with HADC were re-reported,

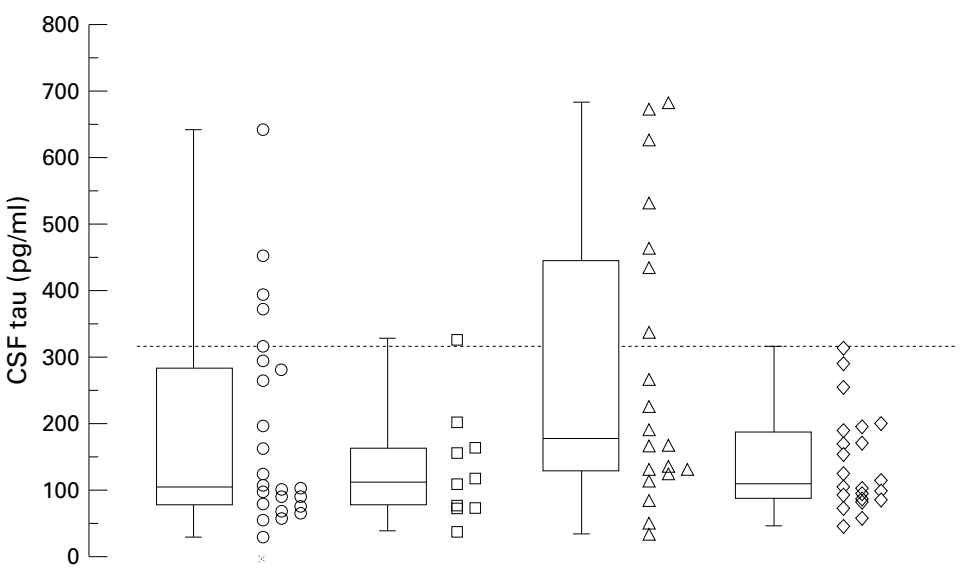

Figure 1 Combined scatter and box and whisker plots of CSF concentrations of tau in patients with HIV associated dementia complex (0) lymphoma () CNS infections $(\triangle)$ and miscellaneous conditions $(\diamond)$. The boxes represent the 25th-75th quartile, divided horizontally by the median. The whiskers represent the range and the adjacent scatter plots represent the individual values from which the boxes and whiskers are derived. The horizontal broken line represents the upper limits of normal (315 pg/ml). mixed in with scans from patients matched for CD4+ T lymphocyte count who had also presented with neurological symptoms and signs including $\mathrm{HADC}$, by a radiologist $(\mathrm{MH}-\mathrm{C})$ experienced in the interpreting of cranial MR images of patients with HIV infection. The radiologist was blinded to the subjects' clinical diagnoses and also did not know which were included in the tau protein study. Predetermined criteria were used to report the MR images; the degree of atrophy (graded none, mild, moderate, or severe) was recorded as previously described. ${ }^{10} 13$

$\mathrm{CSF}$ samples, frozen at $-20^{\circ} \mathrm{C}$, were transferred to the Institute of Neurology for analysis. CSF samples were coded and all analyses were performed blinded to the clinical diagnosis. CSF tau levels were determined using the Innotest human tau antigen second generation sandwich enzyme linked immunosorbent assay (ELISA) (Immuno-genetics, Ghent, Belgium). ${ }^{14}$ The assay's upper limit of normal in CSF is $315 \mathrm{pg} / \mathrm{ml}$ and has a lower limit of detection of $75 \mathrm{pg} / \mathrm{ml}^{15}$

STATISTICAL ANALYSES

Multiple comparisons of normally distributed continuous variables were compared using a one way analysis of variance with post hoc Bonferroni analysis. Non-parametric data were compared using a two tailed Fisher exact test. A p value of $<0.05$ was considered statistically significant.

\section{Results}

Twenty four patients had HADC, 10 had B cell lymphoma, four had primary CNS lymphoma, and six had systemic lymphoma and were undergoing staging investigations (two of these patients had cerebral lymphoma), 20 patients had cerebral infections, including five with CMV encephalitis, five with VZV (three had meningoencephalitis, one had necrotising herpetic retinopathy, and one had an isolated third nerve palsy), seven with cryptococcal meningitis, two with cerebral toxoplasmosis, and one with progressive mutlifocal leucoencephalopathy. Twenty two patients had miscellaneous conditions, including nine with self limiting headache with fever, three with isolated grand mal fits, two with hysterical paraparesis, and eight had a variety of conditions including a single case each of strabismus, chronic demyelinating polyneuropathy, acute confusional state, central pontine myelinolysis, peripheral neuropathy, weight loss without apparent cause, urethral stricture, and paranasal sinusitis.

Measurements of CSF tau (mean (SD)) were similar in patients with $\mathrm{HADC}=212$ (180) $\mathrm{pg} / \mathrm{ml}$, lymphoma = 135 (84) $\mathrm{pg} / \mathrm{ml}$; cerebral infections $=265(200) \mathrm{pg} / \mathrm{ml}$, and miscellaneous conditions $=143(72) \mathrm{pg} / \mathrm{ml} ; \mathrm{p}$ $=$ NS (fig 1). Sixty two patients had normal concentrations of tau in CSF (fig 1) and 14 had elevated concentrations. The diagnosis, treatment, and outcome of those patients with elevated CSF tau concentrations are given in table 1.

In those patients with HADC, the clinical severity of dementia was mild in 15 patients, 
Table 1 Diagnosis, interventions, and outcome in HIV infected patients with elevated CSF tau

\begin{tabular}{llll}
\hline Diagnosis & $\begin{array}{l}\text { CSF tau } \\
(\text { g } / \mathrm{ml})\end{array}$ & Intervention & Outcome and survival \\
\hline HADC & 316 & None & Alive, $>12$ months \\
HADC & 373 & HAART & Alive, $>9$ months \\
HADC & 396 & HAART & Alive, $>12$ months \\
HADC & 452 & None & Died, $<1$ month \\
HADC & 641 & None & Died, $<1$ month \\
Disseminated extraneural NHL & 328 & Chemotherapy & Died, $<1$ month \\
CMV encephalitis & 530 & Ganciclovir & Died, $<1$ month \\
CMV encephalitis & 629 & Ganciclovir & Died, $<1$ month \\
Necrotising herpetic retinopathy (VZV) & 437 & Aciclovir, cidofovir, foscarnet & Alive, $>12$ months \\
VSV meningoencephalitis & 336 & Aciclovir & Alive, $>12$ months \\
PML & 682 & HAART & Alive, $>15$ months \\
Cerebral toxoplasmosis & 673 & Sulphadiazine/pyrimethamine & Alive, $>6$ months \\
Cryptococcal meningitis & 466 & Amphotericin/flucytosine & Died, $<1$ month \\
Isolated grand mal seizure & 316 & None & Alive, $>12$ months
\end{tabular}

HADC = HIV associated dementia complex; PML = progressive multifocal leucoencephalopathy; VZV = varicella zoster virus; $\mathrm{CMV}=$ cytomegalovirus; $\mathrm{NHL}=\mathrm{B}$ cell non-Hodgkin's lymphoma; HAART $=$ highly active antiretroviral therapy.

moderate in six patients, and severe in three patients. Cranial MRI showed atrophy was mild in 12 patients, moderate in five patients, and severe in two patients; five patients had no atrophy. An elevated CSF tau was found in five patients with HADC (table 1). Of these patients three had mild dementia/atrophy, one had moderate dementia/atrophy, and one had severe dementia/atrophy. There was no correlation between the clinical severity of dementia or the degree of atrophy on MRI and the concentration of tau in CSF

Eight patients died within 4 weeks of lumbar puncture and CSF tau measurement; six of these patients had an elevated CSF tau (table 1) $p=0.0024$, two tailed Fisher exact test. Of the eight who died, two with a diagnosis of disseminated lymphoma and normal CSF tau values underwent necropsy which confirmed the antemortem diagnosis. Irrespective of their diagnoses, there was no significant correlation between CD4 + T lymphocyte counts and concentrations of tau in CSF $\left(r^{2}=0.033, \mathrm{p}=\right.$ $0.11)$.

\section{Discussion}

In this study we sought to measure CSF tau in consecutive HIV infected patients undergoing investigation for possible neurological disease. There were three striking findings in the present study. Firstly was the observation that CSF tau was not elevated in the majority $(62 / 76)(82 \%)$ of patients, regardless of their CD4 lymphocyte count and their clinical diagnoses. These data suggest no role for measurement of CSF tau in the diagnosis of HIV infected patients with neurological episodes. These data are similar to those from a smaller study of 32 HIV infected patients of whom nine had HIV associated minor cognitive motor disorder or HADC and 23 were neurologically asymptomatic ${ }^{9}$ : CSF tau was normal in 30/32 (93\%) patients.

The second striking finding in our study was that although $5 / 24$ of those with HADC had elevated CSF levels of tau, there was no correlation between the tau levels and the degree of clinical dementia or atrophy on magnetic resonance imaging. This suggests that CSF tau is a marker of acute neurodestruction and is unlikely to be useful as a marker of chronic low grade neurodestruction in which axonal dam- age $^{16}$ and subsequent release of tau is too low to be detected above the baseline. CSF tau may also be a bulk marker-that is, it is released in proportion to axonal bulk. Thus CSF tau levels may be low in patients with significant axonal loss, provided there is no superimposed acute axonal injury. This may explain the normal CSF tau values in patients with HADC and severe atrophy in whom there is active or ongoing neuroaxonal loss. A simple analogy would be the skeletal muscle isoform of creatine kinase, which is released in proportion to muscle bulk. However, when there is severe muscle wasting - for example, in chronic pyomyositis, active muscle disease may not be associated with an increase above normal, in the level of creatine kinase in blood.

The third striking observation in this study was the finding of elevated CSF tau in 14/76 (18\%) patients and the association between elevated CSF tau and poor survival. Of those with diagnoses other than HADC, elevated CSF tau was seen in conditions causing cerebral necrosis-for example, viral encephalitis due to CMV or VZV and cerebral toxoplasmosis. It is tempting to suggest that the five patients with HADC and elevated CSF tau neuropathologically might have had a more encephalitic process. We are unable to confirm this possibility as we do not have necropsy data on this subgroup of patients.

In conclusion, CSF tau levels were not elevated in the majority of HIV infected patients presenting with acute neurological episodes. In those with HADC, there was no correlation between CSF tau and the degree of dementia or atrophy on magnetic resonance imaging. Although elevated CSF tau was observed in some patients with conditions associated with cerebral necrosis, this finding did not delineate underlying pathology but was associated with a poor outcome.

We thank Professor MJG Harrison for helpful discussion, Drs I G Williams and A Zumla for allowing us to study their patients, and Louise Hinds for typing the manuscript.

Contributors: All authors contributed to the design of the study, to data collection and analysis, and to writing drafts of the manuscript. AJEG performed the tau analyses; $\mathrm{MAH}-\mathrm{C}$ reported the MR images; RFM collated clinical neurochemical reported and imaging data; EJTand GG interpreted the tau measureAJEG, and RFM.

Conflict of interest: None.

Conflict of interest: Nil
Source of funding: Nil 
1 Goedert M, Crowther RA, Garner CC. Molecular characterisation of microtubule-associated proteins tau and MAP2. Tronds Nenri 1991;14:193-9.

2 Arai $H$, Terajima $M$, Miura $M$, et al. Tau in cerebrospinal fluid: a potential marker in Alzheimer's disease. Ann Neurol 1995;38:649-52

3 Oto M, Wiltfang J, Tumani $\mathrm{H}$, et al. Elevated levels of tau protein in cerebrospinal fluid of patients with CreutzfeldtJakob disease. Neurosci Lett 1997;225:210-12.

4 Galasko D, Clark L, Chang L, et al. Assessment of CSF levels of tau protein in mildly demented patients with Alzheimer's disease. Neurology 1997;48:632-5.

5 Arai H, Morikawa Y, Higuchi M, et al. Cerebrospinal fluid tau levels in neruodegenerative diseases with distinct tau-related pathology. Biochem Biophys Res Comm 1997;236:262-4.

6 Harrison MJG, Newman SP, Hall-Craggs MA, et al. Evidence of CNS impairment in HIV infection: clinical, neuro-psychological, EEG, and MRI/MRS study. $\mathcal{f}$ Neurol Neurosurg Psychiatry 1998;65:301-7.

7 Everall IP, Glass JD, McArthur J, et al. Neuronal density in the superior frontal and temporal gyri does not correlate the superior frontal and temporal gyri does not correlate with the degree of human imunodeficiency virus
dementia. Acta Neuropathol 1994;88:538-44.

dementia. Acta Neuropathol 1994;88:538-44.
8 Stanley LC, Mrak RE, Woody RC, et al. Glial cytokines as neuropathogenic factors in HIV infection: pathogenic similarities to Alzheimer's disease. I Neuropathol Exp Neuro 1994;53:231-8

9 Ellis RJ, Seubert P, Motter R, et al. Cerebrospinal fluid tau protein is not elevated in HIV-associated neurologic disease in humans. Neurosci Lett 1998;254:1-4.
10 Miller RF, Lucas SB, Hall-Craggs MA, et al. Comparison of magnetic resonance imaging with neuropathological findings in the diagnosis of HIV and CMV associated CNS disease in AIDS. F Neurol Neurosurg Psychiatry 1997;62: 346-51.

11 Janssen RS, Cornblath DR, Epstein LG, et al. Nomenclature and research case definitions for neurological manifestations of human immunodeficiency virus-type 1 (HIV-1) infection. Neurology 1991;41:778-5.

12 Price RW, Brew BJ. The AIDS dementia complex. F Infect Dis 1988;158:1079-83.

13 Paley MNJ, Chong WK, Wilkinson ID, et al. Cerebrospinal fluid - intracranial volume ratio measurements in patients with HIV infection: CLASS image analysis technique. Radiology 1994;190:879-86.

14 Vandermeeren $M$, Mercken $M$, Vanmechelen $E$, et al. Detection of tau proteins in normal and Alzheimer's disease cerebrospinal fluid with a sensitive sandwich enzyme-linked immunosorbent assay. F Neurochem 1993; 61:1828-34.

15 Green AJE, Harvey RJ, Thompson EJ, et al. Increased tau in the cerebrospinal fluid of patients with fronto-temporal dementia and Alzheimer's disease. Neurosci Lett 1999;259: 133-5.

16 Giometto B, An SF, Groves M, et al. Accumulation of $\beta$-amyloid precursor protein in HIV encephalitis: relationship with neuropsychological abnormalities. Ann Neurol 1997;42:34-40. 